J. Lake Sci. (湖泊科学) , 2015, 27(4):599-606

DOI $10.18307 / 2015.0406$

(C) 2015 by Journal of Lake Sciences

\title{
底泥覆盖对浅水湖泊藻源性湖泛的控制模拟”
}

\author{
商景阁 ${ }^{1,2}$, 何 伟 $^{3}$, 郡世光 $^{4}$,范成新 ${ }^{1 * *}$ \\ ( 1 : 中国科学院南京地理与湖泊研究所湖泊与环境国家重点实验室,南京 210008) \\ (2: 中国药科大学,南京 210009) \\ ( 3 : 上海勘测设计研究院有限公司, 上海 200434) \\ (4:河海大学水文水资源学院, 南京 210098)
}

\begin{abstract}
摘 要: 湖泛的发生与湖泊底部氧化还原条件和致黑致臭物充足供给直接相关. 利用黄土和细沙对太湖湖泛易发区 (月 亮湾) 底泥进行覆盖, 模拟在湖泛可形成条件下, 底泥一水体系及其界面主要物化性质与感官变化过程. 结果表明:0.5 cm 黄土和 $1.0 \mathrm{~cm}$ 细沙的覆盖, 从水色和嗅味半定量角度达到了对湖泛黑臭的控制, 与对照组相比, 覆盖组底泥间隙水中主 要致黑物 $\mathrm{Fe}^{2+}$ 浓度仅为对照组的 1/3, 主要致臭物甲硫醇和二甲基三硫醚等浓度则不到 $50 \%$. 进一步分析底部水体和底 泥性质发现: 经覆盖处理底部水体的溶解氧浓度提高近 1 倍, 氧化还原电位基本处于 $250 \mathrm{mV}$ 以上水平,覆盖层 $1 \mathrm{~cm}$ 左右 表层氧化还原电位和 $\mathrm{pH}$ 均远高于对照底泥. 以黄土为主的底泥覆盖, 主要因阻隔了下层底泥中物质迁移供给和对厌氧 微生物参与的控制, 以及黄土本身性质对湖底物化环境的影响等, 在藻体大量聚集和死亡的水柱环境中, 较好地阻止了 致黑致臭物的形成, 从而较有效控制湖泛的发生.
\end{abstract}

关键词: 湖泛控制;底泥覆盖; 氧化还原; $\mathrm{Fe}^{2+}$

\section{Effects of sediment capping to eliminate simulated alage-caused black bloom in eutrophic lakes}

SHANG Jingge ${ }^{1,2}, \mathrm{HE} \mathrm{Wei}^{3}$, SHAO Shiguang ${ }^{4} \&$ FAN Chengxin ${ }^{1}$

(1: State Key Laboratory of Lake Science and Environment, Nanjing Institute of Geography and Limnology, Chinese Academy of Sciences, Nanjing 210008, P. R. China)

(2: China Pharmaceutical University, Nanjing 210009, P. R. China)

(3: Shanghai Investigation and Design Institute Co. , Ltd. , Shanghai 200434, P. R. China)

(4: College of Hydrology and Water Resources, Hohai University, Nanjing 210098, P. R. China)

Abstract : The occurrence of black bloom is directly related to adequate supply of odor and black substance and redox conditions at the bottom of lakes. Capping with losses and sand was conducted with the sediment from Yueliang Bay, which was heavily polluted and suffered from black blooms in the past years, to investigate the efficiency of black bloom control. The main characteristics of sediment-water system and sensory change process were analyzed. The results shows that capping with losses (0.5 $\mathrm{cm})$ and sand $(1 \mathrm{~cm})$ control the formation of black bloom, from the perspective of visual and olfactory results ( semi-quantitative). The main black substance $\left(\mathrm{Fe}^{2+}\right.$ ) and odor substance (methanethiol and dimethyl trisulfide), in the pore-water of capping sediments was only $1 / 3$ and $1 / 2$ of the control cores, DO concentration and Eh of the bottom water was enhanced up to $100 \%$ and to $250 \mathrm{mV}$, respectively. Eh and $\mathrm{pH}$ of the surface capping sediment was also higher than uncapping control cores. Overall, the integrated capping system could eliminate the diffusion of nutrients from sediment and anaerobic microorganisms in the water, block the formation of taste and odors in the water, and prevent the occurrence of black bloom.

Keywords: Black bloom control; sediment capping; oxidation-reduction; ferrous

* 太湖水污染治理专项( TH2013214)、国家水体污染控制与治理科技重大专项(2012ZX07103-005)和中国科学院南 京地理与湖泊研究所 “一三五” 战略发展规划项目 (NIGLAS2012135008) 联合资助. 2015 - 01 - 29 收稿; 2015 03 - 13收修改稿. 商景阁(1985 ), 男, 博士;E-mail : shangjingge@163.com.

** 通信作者;E-mail:cxfan@ niglas. ac. cn. 
自 2007 年太湖发生的湖泛问题 ${ }^{[-2]}$, 研究者们对富营养化问题的认识上遇到了新挑战, 对湖泊水环境 灾害控制上提出新要求. 相关研究表明, 湖泛的发生除需要藻体或植物体外, 足够面积的污染底泥分布 ${ }^{[3]}$ 和 适宜的环境条件如温度等 ${ }^{[4]}$, 也是其发生的必要条件. 因此只要具备相关的物质和环境条件, 就有可能存在 湖泛发生的风险; 对发生风险的物质和环境条件人为控制的影响作用越大, 湖泛发生的机会就会越低.

在太湖,已启用的湖泛治理方式主要有工程疏浚 ${ }^{[3,5-6]}$ 、蓝藻打捞 ${ }^{[1,7]}$ 以及机械曝气等. 对太湖月亮湾、八 房港、闾江口等不同疏浚区沉积物的模拟研究发现, 疏浚有效抑制湖泛的发生 ${ }^{[3,6]}$; 通过从湖体直接打捞去 除耗氧生物体 (如藻类), 以及水底曝气强化水体复氧等, 也可缓解局部区域的湖泛形成风险 (本课题组未发 表研究结果). 目前在太湖采用的湖泛控制措施, 均是直接或间接改善湖底环境, 即使进行藻类打捞的操作 水层是在近表层, 其主要目的也是为降低堆积于沉积物表层的死亡藻体的量. 已有研究表明, 太湖藻、草等 生源性湖泛的发生与沉积物一水界面物化环境的激烈变化有密切关系 ${ }^{[1-2]}$, 致黑组分 (铁、硫等物质) 和甲基 含硫嗅味物质需要在缺氧和厌氧条件下形成 ${ }^{[8-9]}$, 其物质供给也多来自底部 ${ }^{[10]}$, 因此改善沉积物一水界面物 化环境、阻隔来自底部促进黑臭发生的物质供给,成为控制湖泛的一种可能途径.

在污染河道和湖泊的治理中, 使用天然 (人工) 材料的底泥覆盖技术已经得到广泛的应用并取得了较好 的控制效果 ${ }^{[11-13]}$, 如 Pan 等 ${ }^{[14]}$ 研究发现, 使用土壤及细沙覆盖能够有效控制沉积物总磷、氨氮等向上覆水释 放, 并发现利用较薄厚度 $(1 \mathrm{~cm})$ 的改性细沙覆盖, 就能够有效抑制沉积物的再悬浮, 降低浊度, 即使在受到 一定的扰动作用 (200 转/min) 后, 仍能保持较高的透明度. 鉴于材料覆盖具有阻隔界面两边介质和改变界 面附近物化环境的功能, 本文拟通过对底泥的材料覆盖培养实验, 研究底泥覆盖对因藻类死亡分解导致的 藻源性湖泛的控制作用,探讨其作为湖泛控制的可行性.

\section{1 材料与方法}

\section{1 材料和样品}

1.1. 1 覆盖材料 本文选用的覆盖材料有 2 种, 即黄土和细沙. 黄土取自太湖梅梁湾东岸三国城周边 (去除 $50 \mathrm{~cm}$ 表土后), 风干、研磨过篮(100 目) 备用, 黄土有机碳、总磷、总氮含量分别为 $3.070 、 0.276 、 0.719 \mathrm{~g} / \mathrm{kg}$; 细沙取自梅梁湾东岸砂石场, 将所选细沙用纯水彻底清洗 3 次, 干燥后过 50 目篮备用, 细沙有机碳、总磷、总 氮含量分别为 $0.420 、 0.032 、 0.185 \mathrm{~g} / \mathrm{kg}$.

1.1 .2 底泥底泥采集于太湖北部月亮湾湖区 $\left(31^{\circ} 24^{\prime} 35.64^{\prime \prime} \mathrm{N}, 120^{\circ} 6^{\prime} 3.56^{\prime \prime} \mathrm{E}\right)$, 该区域为太湖湖泛易发区. 使用重力柱状采样器 (内径 $110 \mathrm{~mm}$ ) 采集泥柱高度大于 $25 \mathrm{~cm}$ 的柱状底泥, 垂直放置运送至实验室备用. 底 泥有机碳、总磷、总氮含量分别为 $6.570 、 0.482 、 1.507 \mathrm{~g} / \mathrm{kg}$.

1.1 .3 水样 底泥采集时同步进行, 用有机玻璃采水器采集近底部 $0.5 \mathrm{~m}$ 处上覆水作为实验体系用水. 使用 前经定量滤纸过滤, 去除藻类及悬浮颗粒物.

1.1 .4 藻体 预先于夏季蓝藻暴发期间,在太湖北部用 $25^{\#}$ 浮游生物网采集新鲜藻体,去水后冷冻保存.

\section{2 实验设计及样品分析}

1.2. 1 实验设计 参照已有的研究 ${ }^{[14-15]}$, 将总覆盖厚度设置为 $1.5 \mathrm{~cm}$, 即首先在 3 份平行的柱状底泥表层均 匀覆盖 $0.5 \mathrm{~cm}$ 黄土, 待黄土沉降完全后再均匀覆盖 $1 \mathrm{~cm}$ 细沙, 上层覆沙的目的是抑制底泥的再悬浮 ${ }^{[14]}$. 覆 盖后稳定 $24 \mathrm{~h}$, 再插人间隙水取样装置 (peeper), 并在覆盖层上投加定量藻体 ( $5000 \mathrm{~g} / \mathrm{m}^{2}, 47.5 \mathrm{~g} /$ 柱), 利用 $\mathrm{Y}$ 型再悬浮发生装置模拟湖泛发生所需基本条件 ( $\mathrm{Y}$ 装置详细介绍见 $\mathrm{He}^{\text {等 }}{ }^{\left[{ }^{3]}\right.}$ 及刘国锋等研究成果 ${ }^{[5]}$ ), 设 置环境温度为 $25^{\circ} \mathrm{C}$, 实验过程中每日模拟风浪持续过程 $4 \mathrm{~h}(10: 00-14: 00)$, 整个过程无外加光源并保持自 然光照, 同时设置 1 组无覆盖对照组同步实验.

1.2.2 上覆水感官分析 湖泛的发生过程和程度, 主要是通过人们视觉和嗅觉感官定性得到实时反映. 我们 将肉眼对实验 $Y$ 装置中下部观察到水色分为 4 个等级 $(0 \sim 3): 0$ 为正常湖水或颜色未变化; 1 为浅灰; 2 为 深灰; 3 为黑色 (湖泛发生). 对于嗅味程度的定性判断, 则用烧杯取水样约 $100 \mathrm{ml}$, 通过侧向轻闻按照由轻到 重也为 4 个等级 $(0 \sim 3): 0$ 为无味或正常水体; 1 为微臭; 2 为较臭, 可闻明显臭味; 3 为恶臭. 通过以上视觉 和嗅觉半定量地分析湖泛发生程度. 每天定时采集 1 次样品进行感官分析, 待对照组发生黑臭后再持续 $4 \mathrm{~d}$ 停止. 
1.2 .3 上覆水物化分析 实验开始后, 每天定时取样测定水体溶解氧 ( DO) 、氧化还原电位 ( Eh)、 $\mathrm{pH}$ 、嗅味物 质等指标. DO、Eh、pH 采用手持便携式电极测定; 水样经 $0.45 \mu \mathrm{m}$ 滤膜过滤后, 使用纳氏试剂比色法和钼锑 抗分光光度法分别测定铵态氮 $\left(\mathrm{NH}_{4}^{+}-\mathrm{N}\right)$ 、磷酸盐 $\left(\mathrm{PO}_{4}^{3-}-\mathrm{P}\right)$. 嗅味物质的分析采用顶空固相微萃取 (HSSPME) 气象色谱法 (Agilent 7890A, 配置 FPD 检测器) 进行. 固相萃取条件: 使用二乙烯基苯/Carboxen/聚二 甲基硅氧烷固相微萃取萃取头 (DVB/CAR/PDMS, Supelco, USA), 工作温度 $65^{\circ} \mathrm{C}$, 搅拌速率 500 转/ $\mathrm{min}$, 萃 取 $30 \mathrm{~min}$ 后将萃取头在 GC 进样口 $\left(250^{\circ} \mathrm{C}\right.$ ) 解吸 $3 \mathrm{~min}$. 气相色谱条件: $\mathrm{He}$ 为载气 (流速 $3 \mathrm{ml} / \mathrm{mim}$, 纯度 $0.99999), \mathrm{H}_{2}(50 \mathrm{ml} / \mathrm{min})$ 和 $\mathrm{O}_{2}(65 \mathrm{ml} / \mathrm{min})$ 助燃, $\mathrm{N}_{2}$ 尾吹 $(30 \mathrm{ml} / \mathrm{min}), \mathrm{FPD}$ 检测器温度为 $250^{\circ} \mathrm{C}$, 色谱柱初 始温度为 $45^{\circ} \mathrm{C}$ ( 保持 $5 \mathrm{~min}$ ), 以 $25^{\circ} \mathrm{C} / \mathrm{min}$ 速率升温至 $250^{\circ} \mathrm{C}$ 后保持 $8 \mathrm{~min}$.

1.2. 4 表层微电极分析 待模拟实验结束后, 用 Unisense 微电极 (Unisense, Denmark) 测定沉积物剖面 $\mathrm{Eh}$ 、 $\mathrm{pH}$. 微电极具有检测下限低、空间精度高(可达微米尺度且不破坏界面微环境)、响应速度快 ( $90 \%$ 响应时 间 $<3 \mathrm{~s}, \mathrm{O}_{2}$ 微电极最快响应时间为 $50 \mathrm{~ms}$ ) 等优点. 测定剖面特征时, 将微电极安装在微控制器上, 利用放大 镜裸眼确定界面位置后, 控制器步长设置为 $50 \mu \mathrm{m}$, 获得微米尺度上沉积物 $\mathrm{pH}$ 、 Eh 的剖面特征.

1.2.5 间隙水分析 待沉积物剖面测定后, 取出 peeper 测定间隙水中 $\mathrm{Fe}^{2+} 、 \mathrm{NH}_{4}^{+}-\mathrm{N} 、 \mathrm{PO}_{4}^{3-}-\mathrm{P}$ 浓度. 沉积物中 $\mathrm{Fe}^{2+}$ 与 $\mathrm{Fe}^{3+}$ 浓度采用草酸-草酸铵法测定 ${ }^{[16]}$ : 称取 $0.2 \mathrm{~g}$ 不同层位的新鲜沉积物于 $50 \mathrm{ml}$ 血清瓶中, 加人 $10 \mathrm{ml}$ 充分充氮除氧的草酸一草酸铵溶液, 向血清瓶中冲氮气 $(30 \mathrm{~s})$ 再振荡提取 $16 \mathrm{~h}$ 后比色测定.

\section{2 结果与讨论}

\section{1 底泥覆盖对水体感官的影响}

湖泛最主要的特征就是视觉上的黑与嗅觉上的臭,鉴于视觉上的判断更直接、简单, 因此常将水体颜色 变黑与否作为判断湖泛是否发生的依据 ${ }^{[3,9]}$. 在满足藻源性湖泛发生条件下, 覆盖总厚度 $1.5 \mathrm{~cm}$ 的黄土和 细沙层, 获得与对照差别极大的半定量感官结果. 从水色方面分析 (表 1 ), 对照组在实验第 $7 \mathrm{~d}$ 水体呈浅灰, 第 $8 \mathrm{~d}$ 水体颜色至深灰,第 $10 \mathrm{~d}$ 彻底变黑; 而 3 份平行的覆盖组水体一直到实验结束都没有出现发黑现象, 只是在第 $10 \mathrm{~d}$ 或 $13 \mathrm{~d}$ 出现浅灰色,但未形成湖泛(黑色).

从嗅味方面分析 (表 1), 对照组在第 $5 \mathrm{~d}$ 就开始散发微臭,到第 $7 \mathrm{~d}$ 和第 $8 \mathrm{~d}$ 分别达到较臭和恶臭程度, 并且强烈恶臭持续到实验结束; 而覆盖组水体不仅出现臭味的时间较对照组推迟了 $2 \sim 3 \mathrm{~d}$, 而且散发的臭 味也较轻(微臭).

表 1 湖泛覆盖控制模拟实验中水体的水色和嗅味半定量变化

Tab. 1 Changes in water color and odor during the experiment

\begin{tabular}{|c|c|c|c|c|c|c|c|c|c|c|c|c|c|c|}
\hline \multirow{2}{*}{ 感官 } & \multirow{2}{*}{ 实验组 } & \multicolumn{13}{|c|}{ 时间/d } \\
\hline & & 1 & 2 & 3 & 4 & 5 & 6 & 7 & 8 & 9 & 10 & 11 & 12 & 13 \\
\hline \multirow{4}{*}{ 水色 } & 对照组 & 0 & 0 & 0 & 0 & 0 & 0 & 1 & 2 & 2 & 3 & 3 & 3 & 2 \\
\hline & 覆盖组 1 & 0 & 0 & 0 & 0 & 0 & 0 & 0 & 0 & 0 & 0 & 0 & 0 & 1 \\
\hline & 覆盖组 2 & 0 & 0 & 0 & 0 & 0 & 0 & 0 & 0 & 0 & 1 & 1 & 1 & 1 \\
\hline & 覆盖组 3 & 0 & 0 & 0 & 0 & 0 & 0 & 0 & 0 & 0 & 1 & 1 & 1 & 1 \\
\hline \multirow[t]{4}{*}{ 嗅味 } & 对照组 & 0 & 0 & 0 & 0 & 1 & 1 & 2 & 3 & 3 & 3 & 3 & 3 & 3 \\
\hline & 覆盖组 1 & 0 & 0 & 0 & 0 & 0 & 0 & 1 & 1 & 1 & 1 & 1 & 1 & 1 \\
\hline & 覆盖组 2 & 0 & 0 & 0 & 0 & 0 & 0 & 1 & 1 & 1 & 1 & 2 & 1 & 1 \\
\hline & 覆盖组 3 & 0 & 0 & 0 & 0 & 0 & 0 & 0 & 0 & 0 & 0 & 0 & 1 & 1 \\
\hline
\end{tabular}

从水色及嗅味变化结果来看, 覆盖处理在降低水体发臭程度的同时, 也抑制了水体致黑物质的产生, 有 效降低湖泛的发生风险.

\section{2 底泥覆盖对水体 DO、Eh 和 $\mathrm{pH}$ 的影响}

当加人藻体后, 泥一水一藻对照组体系的 DO、Eh 和 $\mathrm{pH}$ 均出现大幅度下降, 而覆盖组除 DO 外, 其他下降 不太明显, 甚至 $\mathrm{Eh}$ 和 $\mathrm{pH}$ 在覆盖初期的 $0 \sim 6 \mathrm{~d}$ 还出现小幅上升, 相应数值绝大多数也高于对照组(图 1). 分 
析稳定阶段上覆水 DO 浓度, 对照组平均值为 $0.7 \sim 1.0 \mathrm{mg} / \mathrm{L}$, 处于厌氧环境, 而覆盖组平均值几乎提高 1 倍, 达到 $2 \mathrm{mg} / \mathrm{L}$ 左右. 尽管在模拟实验后期, 覆盖组 $\mathrm{Eh}$ 有一定程度的下降, 但总体上覆盖组 $\mathrm{Eh}$ 要远高于对 照组.
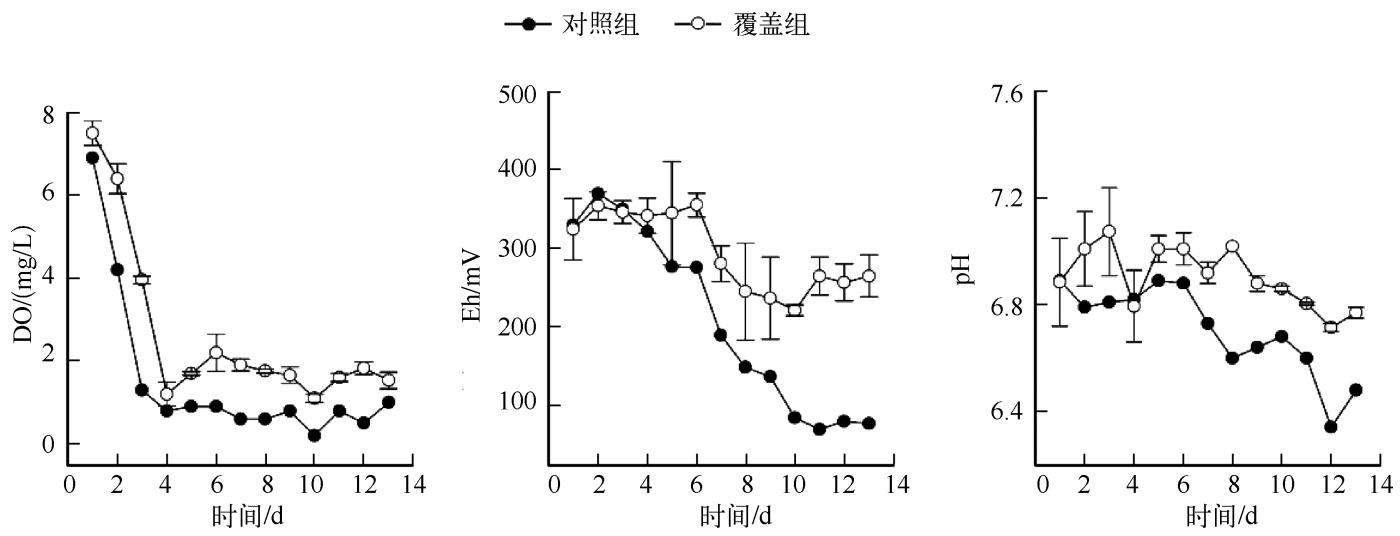

图 1 底泥覆盖对水体 DO、Eh、pH 的影响

Fig. 1 The effect of sediment capping on DO, Eh and $\mathrm{pH}$ of overlying water

覆盖改善了水体的氧化还原条件. 在沉积物一水体系中, $\mathrm{O}_{2}$ 等电子受体的消耗主要有沉积物耗氧 (有机 质矿化、微生物呼吸等) 及藻类腐烂分解等途径 ${ }^{[17]}$. 本研究选用的覆盖材料 (黄土、细沙) 有机碳含量低于沉 积物, 且相对难以被微生物利用矿化. 覆盖于污染沉积物表层的土和沙, 利用黏土成分的层阻能力, 使其在 抑制沉积物表层耗氧速率 (SOD) 的同时, 也有效抑制沉积物中污染物向上覆水的扩散和阻止底泥中微生 物, 如梭菌 ${ }^{[18]}$ 和硫酸还原菌 ${ }^{[19]}$ 与残藻生物质的接触, 抑制藻体的腐烂分解进程和氧气消耗速率, 从而控制 DO 浓度降低,改善培养体系的氧化还原条件.

周芗等 ${ }^{[20]}$ 通过在高度厌氧底泥上进行土壤和硅藻土原位覆盖修复, 发现覆盖 $1 \mathrm{~cm}$ 厚度的材料就能够 隔绝污染戻氧底泥与水体, 大幅提高底泥的 $\mathrm{Eh}$, 明显逆转沉积物底质和水质. 当体系的 $\mathrm{Eh}$ 与 $\mathrm{pH}$ 处于较低 的水平, $\mathrm{FeS}$ 和甲基硫醚类物质等特征污染物更容易产生和稳定累积 ${ }^{[10,21]}$, 覆盖处理一定程度上使得水底氧 化还原条件得到改善, 这对于湖泛的控制起到积极作用. 因此底泥土沙覆盖处理在抑制沉积物 $\mathrm{Eh} 、 \mathrm{pH}$ 显著 降低的同时,也抑制了湖泛易发区的黑臭发生风险.

\section{3 底泥覆盖对典型嗅味物质的抑制作用}

覆盖处理能有效抑制水体中甲基含硫嗅味物质的增加与累积 (图 2). 对照组嗅味物质浓度在实验第 $9 \mathrm{~d}$ 急剧增加, 而覆盖处理组在整个实验周期内 $(13 \mathrm{~d})$ 变化趋缓, 尽管在实验后期, 嗅味物质浓度有小幅增加, 但却远远小于对照组.

Yang 等 ${ }^{[22]}$ 通过对 2007 年无锡水危机样品的分析认为, 浓度高达 $11399 \mathrm{ng} / \mathrm{L}$ 的二甲基三硫醚( DMTS) 是湖泛水体产生臭味的主要原因, 其来源可能与藻类死亡分解产生的含硫氨基酸一甲基氨酸和半胱氨酸 的微生物戻氧分解过程等有关 ${ }^{[21]}$. 含硫嗅味物质广泛存在于自然环境中, 在海洋 ${ }^{[23]}$ 、河道 ${ }^{[24]}$ 以及湖泊 (水 库 $)^{[9]}$ 中均有相关的报道. 水体中含硫嗅味物质的来源比较广泛, 在某些特定的条件下, 来源于藻类、动植物 残体的各种有机含硫的前驱物能够通过不同的降解途径转化为含硫嗅味物质. 在淡水水体以及缺氧沉积物 中, 含硫嗅味物质主要通过硫化物的甲基化途径生成 ${ }^{[8]}$, 因此氧化还原条件是决定湖泛水体臭味产生和持 续累积的关键因子. 而覆盖处理通过提高沉积物表层的氧化还原环境, 阻隔沉积物一水之间的物质交换过 程, 有效抑制水体嗅味物质浓度 ${ }^{[25]}$. 同时低 $\mathrm{pH}-\mathrm{Eh}$ 条件的维持, 也利于微生物对含硫蛋白质的厌氧分解作 用,促进水体中含硫嗅味物质的产生和累积 ${ }^{[21]}$, 促进湖泛的发生和稳定存在.

\section{4 底泥覆盖对间隙水物质浓度分布的影响}

沉积物中 $\mathrm{Fe}^{2+}$ 浓度增加是湖泛发生的主要基本特征和要求之一. 底泥中的 $\mathrm{Fe}^{2+}$ 浓度及 $\mathrm{Fe}^{2+} / \mathrm{Fe}^{3+}$ 的转 换多受体系中 $\mathrm{Eh}$ 的控制, 其存在与浓度大致反映所处环境的氧化还原水平 ${ }^{[26-27]}$. 在厌氧条件的驱动下, 

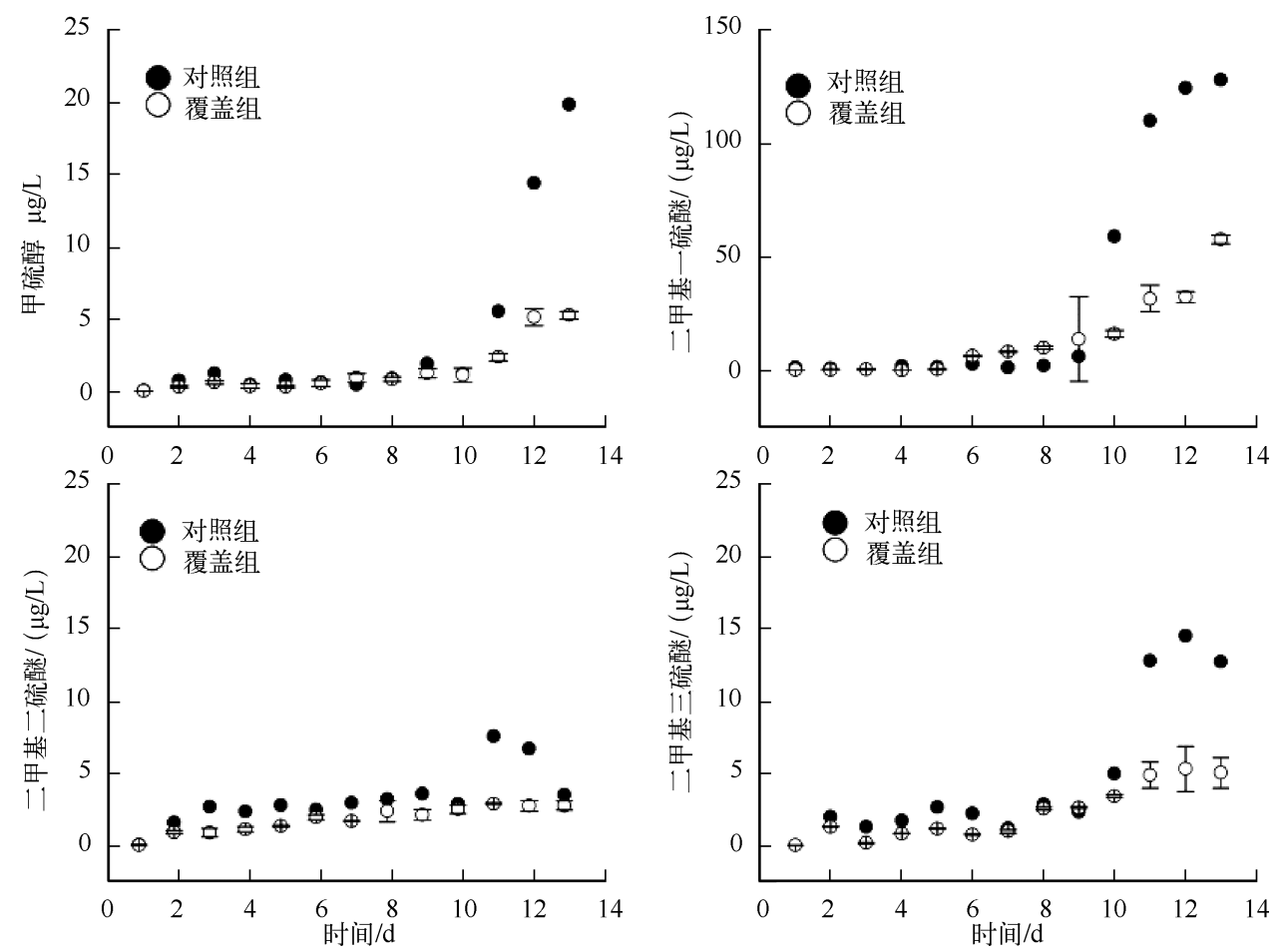

图 2 覆盖状态下典型含硫臭味物质浓度的变化过程

Fig. 2 The effect of sediment capping on concentration of sour odor substance in the overlying water

$\mathrm{Fe}^{3+}$ 向 $\mathrm{Fe}^{2+}$ 的转化导致 $\mathrm{Fe}^{2+}$ 浓度的增加, 因此与覆盖组相比, 发生黑臭的对照组沉积物中 $\mathrm{Fe}^{2+}$ 占总铁的比 例显著增加 (图 3). 从间隙水中 $\mathrm{Fe}^{2+}$ 浓度看, 覆盖处理组沉积物中 $\mathrm{Fe}^{2+}$ 浓度低于对照组, 其平均浓度约为对 照黑臭组的 $1 / 3$, 强还原条件是造成对照组中 $\mathrm{Fe}^{2+}$ 浓度 高于覆盖组的主要原因 (图 4). $\mathrm{Fe}^{2+}$ 浓度增加可能造成 两方面的结果, 首先, 自由铁离子与还原态硫结合成黑 色 $\mathrm{FeS}$ 沉淀,诱发水体发黑 ${ }^{[22,28]}$. 其二, 沉积物中铁形态 的变化 $\left(\mathrm{Fe}^{3+} / \mathrm{Fe}^{2+}\right.$ 转换 $)$ 对控制沉积物中磷的释放具有 重要意义 ${ }^{[29]}, \mathrm{Fe}^{3+}$ 在戻氧条件下向 $\mathrm{Fe}^{2+}$ 的转化造成沉 积物中铁结合态磷的释放,为藻华再次发生提供营养基 础 ${ }^{[2]}$. 因此从实验结果能够推断, 通过对底泥进行覆盖, 可提高体系的 $\mathrm{Eh}$, 降低水体中主要特征污染物浓度, 从 而有效控制湖泛的发生程度.

对照组上覆水及间隙水中 $\mathrm{NH}_{4}^{+}-\mathrm{N} 、 \mathrm{PO}_{4}^{3-}-\mathrm{P}$ 剖面浓 度高于覆盖组 (图 4). 高浓度的 $\mathrm{NH}_{4}^{+}-\mathrm{N} 、 \mathrm{PO}_{4}^{3-}-\mathrm{P}$ 负荷是 湖泛的主要特征 ${ }^{[22,28]}$, 其产生和归趋主要与底泥污染水 平、生物作用大小、氧化还原条件及水动力扰动程度等 多种条件有关 ${ }^{[30]}$. 由于藻类的死亡分解作用降低了水体 及沉积物的 $\mathrm{Eh}$ (图 5), 表层底泥呈缺氧状态, 还原性增 强, 沉积物反硝化和氨化作用较明显, 沉积物中 Fe 结合



图 3 沉积物 $\mathrm{Fe}^{2+}$ 浓度占总铁比例的剖面变化

Fig. 3 Vertical changes of the percentage of $\mathrm{Fe}^{2+}$ concentration to total $\mathrm{Fe}$ in the sediment 
态磷快速释放, 从而提高水体中 $\mathrm{NH}_{4}^{+}-\mathrm{N} 、 \mathrm{PO}_{4}^{3-}-\mathrm{P}$ 负荷. 而实验中使用的覆盖材料有机质及总氮含量极低, 受 覆盖效应、化学效应和物理吸附的影响 ${ }^{[12]}$, 覆盖层既可通过吸附作用阻止间隙水向上覆水的扩散释放,也能 通过改善底部 $\mathrm{Eh}$ 而抑制 $\mathrm{NH}_{4}^{+}-\mathrm{N} 、 \mathrm{PO}_{4}^{3-}-\mathrm{P}$ 浓度的迅速增加 ${ }^{[2]}$. 因此覆盖降低水体营养盐负荷的同时, 也可降 低水华再次暴发的风险.
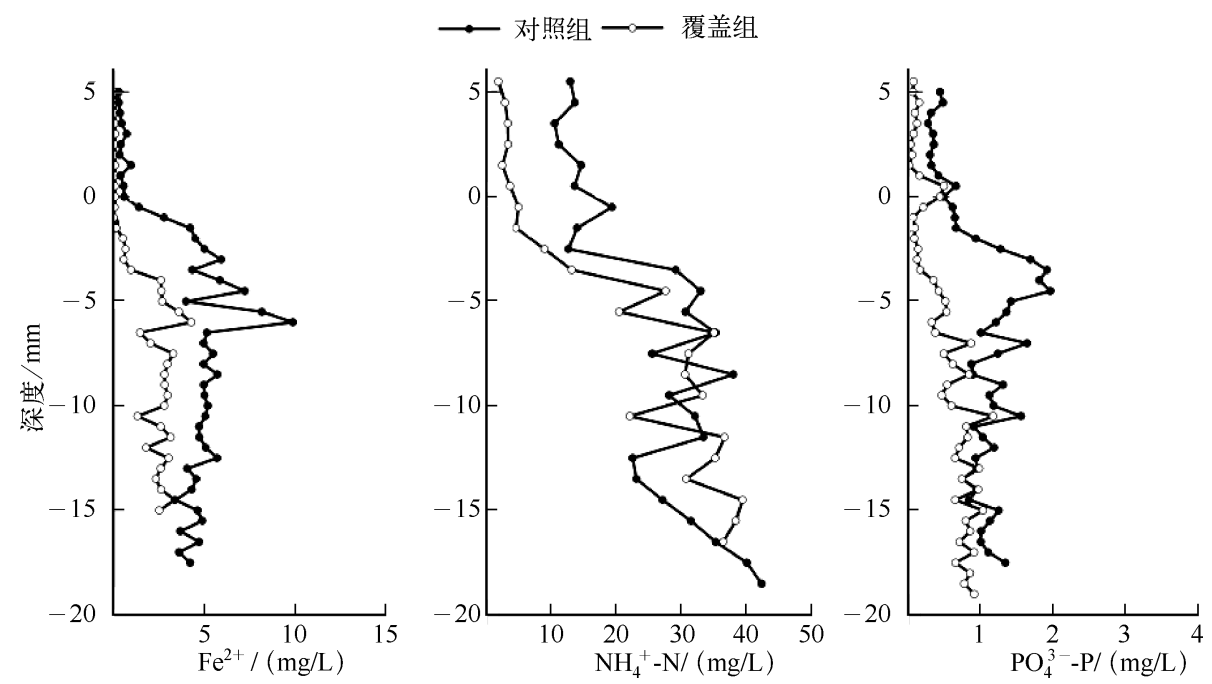

图 4 沉积物间隙水中 $\mathrm{Fe}^{2+}, \mathrm{NH}_{4}^{+}-\mathrm{N}$ 和 $\mathrm{PO}_{4}^{3-}-\mathrm{P}$ 浓度变化

Fig. $4 \mathrm{Fe}^{2+}, \mathrm{NH}_{4}^{+}-\mathrm{N}$ and $\mathrm{PO}_{4}^{3-}-\mathrm{P}$ concentrations in the pore-water of the sediment

\section{5 底泥覆盖对沉积物微界面 Eh、pH 分布的影响}

沉积物的微界面变化特征对分析湖泛致黑与致臭物质的形成有重要意义. 沉积物 $\mathrm{Eh}, \mathrm{pH}$ 剖面分布图可 以看出 (图 5), 不同处理间沉积物 $\mathrm{Eh} 、 \mathrm{pH}$ 剖面存在显著差异. 与覆盖组相比, 对照组 (发生黑臭) Eh 急剧降 低, 上覆水 $\mathrm{Eh}$ 在 $300 \mathrm{mV}$ 左右, 在沉积物界面下 $1 \mathrm{~mm}$ 处急剧降低, 直至沉积物中约 $1.5 \mathrm{~mm}$ 处变化趋缓并稳 定在 $70 \mathrm{mV}$ 左右. 覆盖组水体 $\mathrm{Eh}$ 约 $500 \mathrm{mV}$, 在进人沉积物后仍保持较高水平 (409 mV), 在距界面 $7.5 \mathrm{~mm}$ 深度上, Eh 变化缓慢并趋于稳定, 其稳定深度要远远大于对照. 沉积物 $\mathrm{pH}$ 的变化趋势与 $\mathrm{Eh}$ 类似, 对照组上 覆水、沉积物 $\mathrm{pH}$ 均小于覆盖组, 即湖泛过程降低了体系的 $\mathrm{pH}$. 从沉积物 $\mathrm{Eh} 、 \mathrm{pH}$ 的剖面分布结果来看, 对照 组体系处于还原状态, 而覆盖组则不仅 $\mathrm{pH}$ 值从剖面上一直高于对照组, 而且同一深度的 $\mathrm{Eh}$ 也远高于对 照组.

沉积物-水体系中 $\mathrm{O}_{2} 、 \mathrm{NO}_{3}^{-} 、 \mathrm{Mn}(\mathrm{IV}) 、 \mathrm{Fe}(\mathrm{III}) 、 \mathrm{SO}_{4}^{2-}$ 等电子受体控制着沉积物的 $\mathrm{Eh}$, 而这些电子受体同 时受到有机质降解速率以及微生物等因素的影响 ${ }^{[31]}$, 在某些极端条件, 如藻类大量堆积、死亡分解的过程 中, 电子受体被大量消耗, 导致沉积物中氧化层厚度降低甚至消失, 沉积物的 Eh 也表现出下降的趋势. 从沉 积物间隙水中 $\mathrm{pH}$ 剖面变化曲线可以看出, 与覆盖组 (未黑臭) 相比, 发生黑臭后, 间隙水 $\mathrm{pH}$ 显著下降 (图 5 ). 沉积物中的 $\mathrm{pH}$ 对于体系内离子活度、物质溶解电离平衡、化学反应速率、微生物活性等都具有广泛的影 响. Belzile 等 ${ }^{[26]}$ 和 $\mathrm{He}$ 等 $^{[3]}$ 分别在厌氧和湖泛沉积物中发现了类似的现象, 其原因可能与有机质快速降解 后氧化还原体系的降低以及有机质的矿化等有关, 但仍需进一步研究. $\mathrm{Eh}$ 与 $\mathrm{pH}$ 共同影响众多化学反应的 方向与平衡过程, 推动物质氧化还原状态的转变过程. 而低 $\mathrm{pH}-\mathrm{Eh}$ 环境有利于还原态 $\mathrm{Fe}^{2+}$ 的形成, 促进水 体中无定形 $\mathrm{FeS}$ 的形成且能够稳定存在,是水体发黑的重要诱因之一 ${ }^{[32]}$.

本实验中选用的覆盖材料为有机质含量低的清洁黄土与细沙, 覆盖层耗氧性低, 同时天然黄土中含有 丰富的黏土成分, 对污染物的吸附能力较强. 因此覆盖层的存在阻隔了沉积物与上覆水之间的交换过程, 能 够降低沉积物及微生物耗氧速率, 从而减缓藻类腐烂分解的过程, 维持体系 $\mathrm{Eh}$ 的稳定, 改善沉积物 $\mathrm{pH}-\mathrm{Eh}$ 条件, 对湖泛的控制起到积极的作用. 

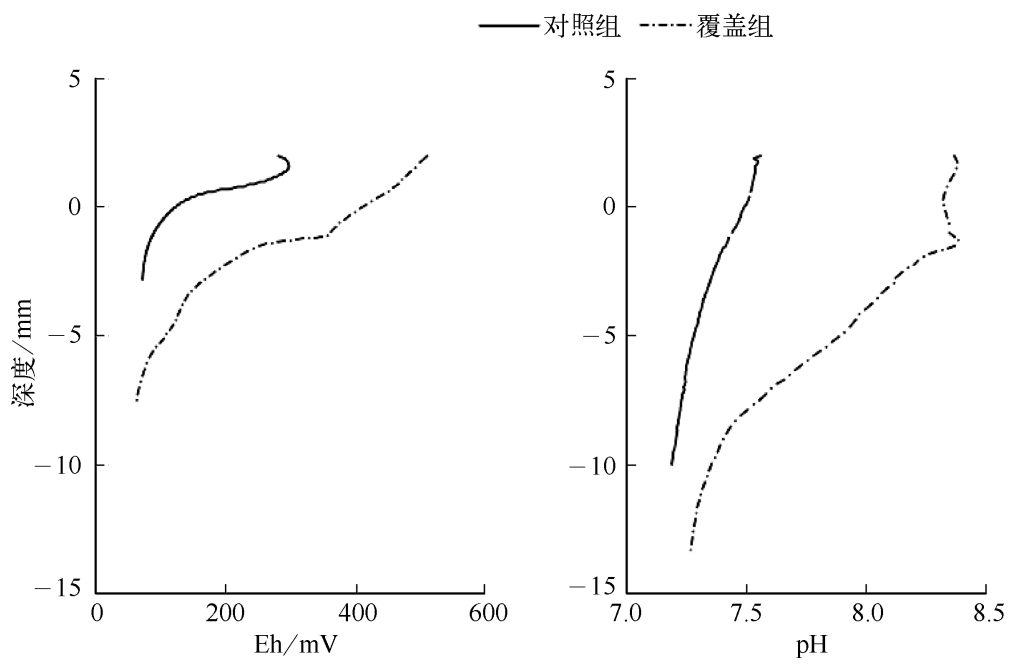

图 5 沉积物 Eh、pH 剖面特征

Fig. 5 Vertical profiles of Eh and $\mathrm{pH}$ in the sediment

\section{3 结论}

覆盖处理水体中 $\mathrm{NH}_{4}^{+}-\mathrm{N} 、 \mathrm{PO}_{4}^{3-}-\mathrm{P} 、 \mathrm{Fe}^{2+}$ 浓度低于未覆盖对照组,4 种甲基含硫臭味物质浓度也得到明显 抑制. 底泥覆盖在改善沉积物氧化还原状态的同时,降低沉积物与上覆水间的交换速率, 有效抑制水体发黑 发臭程度. 结果表明通过在湖泛高风险区进行底泥覆盖, 可有效降低湖泛风险, 因此可将覆盖作为湖泛预控 措施之一. 本研究采用的覆盖厚度为 $1.5 \mathrm{~cm}$, 理论上随着覆盖厚度增加, 其控制效果也会更加显著, 但实际 操作工程量也必将随覆盖厚度增加而倍增, 因此如何通过强化覆盖材料的控制效果来降低覆盖厚度尚需要 进一步研究.

致谢: 中国科学院太湖湖泊生态系统研究站在样品采集中提供了帮助, 申秋实博士、何翔博士在实验过程中 给与了重要帮助, 特此感谢!

\section{4 参考文献}

[1 ] 陆桂华,马 倩. 太湖水域“湖泛”及其成因研究. 水科学进展, 2009, 20(3)：438-442.

[2] 刘国锋,何 俊,范成新等. 藻源性黑水团环境效应: 对水一沉积物界面处 Fe, Mn, S 循环影响. 环境科学, 2010, 31(11) : 2652-2660.

[ 3 ] He W, Shang J, Lu X et al. Effects of sludge dredging on the prevention and control of algae-caused black bloom in Taihu Lake, China. Journal of Environmental Sciences, 2013, 25(3) : 430-440.

[ 4 ] 王成林,张 咏,张宁红等. 太湖藻源性“湖泛”形成机制的气象因素分析. 环境科学, 2011, 32(2): 401-408.

[5] 刘国锋. 藻源性湖泛对太湖沉积物一水界面物质行为影响及预控研究 [学位论文]. 南京: 中国科学院南京地理与湖 泊研究所, 2009 .

[6] 陈 超,钟继承,范成新等. 疏浚对湖泛的影响: 以太湖八房港和问江口水域为例. 中国环境科学, 2014, 34(8): 2071-2077.

[ 7 ] 韩士群,严少华,王震宇等. 太湖蓝藻无害化处理资源化利用. 自然资源学报, 2009, 24(3): 431-438.

[ 8 ] Stets EG, Hines ME, Kiene RP. Thiol methylation potential in anoxic, low-pH wetland sediments and its relationship with dimethylsulfide production and organic carbon cycling. FEMS Microbiology Ecology, 2004, 47(1):1-11.

[ 9 ] Lu X, Fan C, Shang J et al. Headspace solid-phase microextraction for the determination of volatile sulfur compounds in odorous hyper-eutrophic freshwater lakes using gas chromatography with flame photometric detection. Microchemical Journal, 2012, 104 : 26-32.

[10］申秋实,邵世光,王兆德等. 风浪条件下太湖藻源性“湖泛” 的消退及其水体恢复进程. 科学通报, 2012, 57 (12)： 
1060-1066.

[11] Jacobs PH, Förstner U. Concept of subaqueous capping of contaminated sediments with active barrier systems (ABS) using natural and modified zeolites. Water Research, 1999, 33(9) : 2083-2087.

[12] 叶恒朋,陈繁忠, 盛彦清等. 覆盖法控制城市河涌底泥磷释放研究. 环境科学学报, 2006, 26(2) : 262-268.

[13] Förstner U, Apitz SE. Sediment remediation: US focus on capping and monitored natural recovery. Journal of Soils and Sediments, 2007, $7(6):$ 351-358.

[14] Pan G, Dai L, Li L et al. Reducing the recruitment of sedimented algae and nutrient release into the overlying water using modified soil/sand flocculation-capping in eutrophic lakes. Environmental Science and Technology, 2012, 46 (9): 5077-5084.

[15] Sun H, Xu X, Gao G et al. A novel integrated active capping technique for the remediation of nitrobenzene-contaminated sediment. Journal of Hazardous Materials, 2010, 182(1) : 184-190.

[16] Phillips EJ, Lovley DR. Determination of Fe ( III ) and Fe ( II ) in oxalate extracts of sediment. Soil Science Society of America Journal, 1987, 51(4) : 938-941.

[17 Z Zhang L, Shen Q, Hu H et al. Impacts of Corbicula fluminea on oxygen uptake and nutrient fluxes across the sediment-water interface. Water, Air, and Soil Pollution, 2011, 220(1/2/3/4) : 399-411.

[18 ] Xing P, Guo L, Tian W et al. Novel Clostridium populations involved in the anaerobic degradation of Microcystis blooms. The ISME Journal, 2010, 5(5) : 792-800.

[19] Feng Z, Fan C, Huang W et al. Microorganisms and typical organic matter responsible for lacustrine "black bloom". Science of the Total Environment, 2014, 470: 1-8.

[20］周 莹,潘 纲,陈 影. 土壤原位覆盖对底泥的修复作用研究. 环境工程学报, 2011, 5(11): 2459-2463.

[21] 卢 信, 冯紫艳, 商景阁等. 不同有机基质诱发的水体黑臭及主要致臭物 (VOSCs) 产生机制研究. 环境科学, $2012, \mathbf{3 3}(9)$ : 3152-3159.

[22] Yang M, Yu J, Li Z et al. Taihu Lake not to blame for Wuxi's woes. Science, 2008, 319(5860) : 158.

[23] Wakeham SG, Sinninghe Damsté JS, Kohnen ME et al. Organic sulfur compounds formed during early diagenesis in Black Sea sediments. Geochimica et Cosmochimica Acta, 1995, 59(3) : 521-533.

[24] 盛彦清. 广州市典型污染河道与城市污水处理厂中恶臭有机硫化物的初步研究 [学位论文]. 广州: 中国科学院广 州地球化学研究所, 2007.

[25] 李巧霞,潘 纲, 王 丹等. 太湖十八湾嗅味物质变化规律及除嗅方法. 环境工程学报, 2012, 6(9): 3191-3195.

[26] Belzile N, Pizarro J, Filella M et al. Sediment diffusive fluxes of Fe, Mn, and P in a eutrophic lake: Contribution from lateral vs bottom sediments. Aquatic Sciences, 1996, 58(4) : 327-354.

[27] 李 真,黄民生,何 岩等. 铁和硫的形态转化与水体黑臭的关系. 环境科学与技术, 2010, 33(6): 1-7.

[28 ] Shen Q, Liu C, Zhou Q et al. Effects of physical and chemical characteristics of surface sediments in the formation of shallow lake algae-induced black bloom. Journal of Environmental Sciences, 2013, 25(12) : 2353-2360.

[29] 王晓蓉,华兆哲. 环境条件变化对太湖沉积物磷释放的影响. 环境化学, 1996, 15(1): 15-19.

[30］范成新,杨龙元. 太湖底泥及其间隙水中氮磷垂直分布及相互关系分析. 湖泊科学, 2000, 12 (4) : 359-366.

[31] 王敬富, 陈敬安, 曾 艳等. 微电极测量系统在湖泊沉积物一水界面生物地球化学过程研究中的应用. 地球与环境, $2013,41(1)$ : $65-70$.

[32］宋金明. 中国近海沉积物一海水界面化学. 地球科学进展, 1998, 13(6) : 590 . 